\title{
Low-field microwave absorption in pulse laser deposited FeSi thin film
}

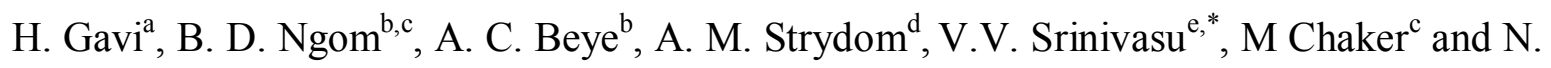
Manyala, ${ }^{\mathrm{a}, *}$

${ }^{a}$ Department of Physics, SARCHI Chair in Carbon Technology and Materials, Institute of Applied Materials, University of Pretoria, Pretoria 0028, South Africa

${ }^{b}$ Groupe d Laboratoires de Physique des Solid et des Matériaux, Faculté deSciences et Techniques, Université Cheikh Anta de Dakar, BP 25114 Dakar-Fann, Daka 16996, Senegal

${ }^{c}$ Institut National de la Recherche Scientifique Centre - Énergie Matériaux

Télécommunications 1650, Boul. Lionel Boulet Varennes (Québec) J3X 1S2

${ }^{d}$ Department of Physics, University of Johannesburg, Johannesburg 2006, South Africa

${ }^{e}$ Department of Physics, University of South Africa, Pretoria 0003, South Africa

\begin{abstract}
Low field microwave absorption (LFMA) measurements at $9.4 \mathrm{GHz}$ (X-band), were carried out on pulse laser deposited (PLD) polycrystalline B20 cubic structure FeSi thin film grown on $\mathrm{Si}(111)$ substrate. The LFMA properties of the films were investigated as a function of DC field, temperature, microwave power and the orientation of DC field with respect to the film surface. The LFMA signal is very strong when the DC field is parallel to the film surface and vanishes at higher angles. The LFMA signal strength increases as the microwave power is increased. The LFMA signal disappears around $340 \mathrm{~K}$, which can be attributed to the disappearance of ferromagnetic state well above room temperature in these films. We believe that domain structure evolution in low fields, which in turn modifies the low field permeability as well as the anisotropy could be the origin of the LFMA observed in these films. The observation of LFMA opens the possibility of the FeSi films to be used as low magnetic field sensors in the microwave and rf frequency regions.
\end{abstract}

Keywords: Pulsed laser ablation, crystalline B20 FeSi film; Low field microwave absorption

*Corresponding authors

E-mail address: ncholu.manyala@up.ac.za (Manyala); vallavs@unisa.ac.za (Srinivasu) 


\section{Introduction}

$\varepsilon$-FeSi is a small band-gap insulator with a cubic B20 (viewed as distorted $\mathrm{NaCl}$ (B1) structure) crystal structure which is the only d electron system classified as Kondo insulator [1-4]. In film form with the same crystal structure synthesized by pulsed laser deposition (PLD) FeSi was observed to make a magnetic transition from paramagnet to ferromagnet [5]. Ferromagnetic materials normally exhibit ferromagnetic resonance (FMR) when exposed to microwaves and appropriate magnetic fields that satisfy resonance conditions. A relatively new phenomenon called low-field microwave absorption (LFMA) - Microwave power absorption (MPA) centered at zero magnetic field $(\mathrm{B}=0)$ has been observed in ferromagnetic materials and various other materials such as high temperature superconductors, ferrites, manganites, semiconductors, doped silicate glasses and in soft magnetic materials [6-11]. Different interpretations and explanations have been put forward to try and explain the appearance of LFMA signal at $\mathrm{B}=0$ in a wide variety of materials. For magnets and ferrites the LFMA signal is associated with the onset of the ordered phase and provides a sensitive detector of magnetic ordering [7, 12, 13]. For high-temperature superconductors LFMA signal reveals a change to superconductive state [6], while in semi-conductors the LFMA signal is due to magneto-resistive effects [13]. For doped silicate glasses LFMA signal is due to magneto-induced microwave conductivity in the dielectric glass which derives from spindependent charge migration within the first co-ordination of paramagnetic ion [14]. For soft magnetic materials the LFMA signal is due to low-field spin magnetization processes [8]. Montiel et al have reported a strong correlation between LFMA and giant magneto impedance (GMI) phenomenon mostly in Co-based amorphous ribbons [8]. By using complex Poynting vector Alvarez et.al [15] showed a theoretical correlation between LFMA and magneto impedance (MI) as given by the following relationship.

$$
\frac{d P_{S}}{d H} \alpha \frac{d R_{e}\left(Z_{S}\right)}{d H}
$$

In this paper we report on the microwave power absorption behavior of crystalline B20 cubic structure FeSi thin film when subjected to microwave magnetic fields at $9.4 \mathrm{GHz}$ (X-band) in the usual electron spin resonance (ESR) configuration (microwave magnetic field normal to DC field). The expectation was to observe high field FMR which is usual for ferromagnetic systems since the films had already been as observed as ferromagnetic [5], but in addition a LFMA signal centered at zero field was also observed. We then performed detailed LFMA 
measurements under different measuring conditions and geometries. It is for the first time that the LFMA phenomenon was reported in the crystalline B20 FeSi in thin film form. This observation makes FeSi magnetic thin films on Si substrate be a potential candidate for low magnetic field sensor in the microwave and rf frequency regions.

\section{Experimental details}

The mixture of high purity Fe (99.999\%) and $\mathrm{Si}(99.999 \%)$ with 50\% - 50\% prescribed ratio was melted by arc melter in an argon atmosphere. Resulting FeSi bulk samples were annealed for 24 hours at $1000^{\circ} \mathrm{C}$ in evacuated quartz ampoules to improve homogeneity. The X-ray diffraction powder analysis showed a cubic B20 crystalline structure. Films were deposited on $\mathrm{Si}$ (111) substrates held at $450{ }^{0} \mathrm{C}$ in a vacuum $\left(10^{-6} \mathrm{Torr}\right)$. Substrates were cleaned ultrasonically with several organic solvents as well as with de-ionized water, etched in a $20 \% \mathrm{HF}$ solution and left in air for a period of between 2 and 30 days prior to deposition. An eximer laser, wavelength of $248 \mathrm{~nm}$, fluence of $4 \mathrm{~J} / \mathrm{cm}^{2}$, repletion rate of $10 \mathrm{~Hz}$, and 30 ns pulse duration was incident on arc melted FeSi target for a period of 15 minutes. The film stoichiometry and thickness was determined by Rutherford backscattering spectroscopy (RBS). $\mathrm{Cu} \mathrm{K \alpha}$ X-ray diffraction (XRD) pattern using an AXS Bruker diffractometer equipped with position sensitive detector determined the crystalline structure of both FeSi bulk and film. The film morphology was characterized via scanning electron microscopy (SEM), while transmission electron microscopy (TEM) probed the cross-sectional microstructure of the film.

Magnetization (M) of the film was measured using quantum design magnetic property measuring system (MPMS) in the DC mode at room temperature. The microwave absorption measurements were made on $200 \mathrm{~nm}$ thick films using JEOL-ESR spectrometer operating at 9.4 GHz (X-band). The film was mounted in the cavity centre at a position where the microwave magnetic field is maximum. The DC static field $H_{D C}$ was slowly swept between 0 Oe and 1500 Oe. It should be noted that even though in the figures we show the field sweep from negative to positive direction of the field (due to instrument computer settings), yet the instrument could not sweep on the negative field side. The microwave power was varied between $3 \mathrm{~mW}$ to $20 \mathrm{~mW}$. The DC field was modulated with a superposed ac field whose amplitude was varied between $1 \mathrm{Oe}$ and $6 \mathrm{Oe}$ at $100 \mathrm{kHz}$ frequency. The microwave response is measured as a derivative signal. The temperature was varied between $293 \mathrm{~K}$ and $370 \mathrm{~K}$. 


\section{Results and discussion}

Fig. 1 show XRD data for both bulk and film samples with all peaks belonging to either the B20 structure or the diamond structure of the Si substrate in case of the film. No evidence for secondary phase for the film has been found in any of the scans indicating that film is likely a single phase as it well compares with the bulk FeSi sample. High resolution TEM image of FeSi film reveals sharp and continuous film-substrate interface, as in Fig. 2 (a) [5]. Fig. 2(b) shows the SEM image which displays almost featureless, extremely smooth film surface that are free of clusters and islands with exception of small density of droplets common to PLD growth (bright features in the figure). The bulk DC magnetization hysteresis measurement at $300 \mathrm{~K}$ is shown in Fig. 3 (a) which clearly shows the film being magnetic even at room temperature even though it has very low moments as compared to measurements at low temperature [5]. Fig.3 (b) Shows the derivative of microwave absorption $(d P / d H)$ vs DC field $\left(\mathrm{H}_{\mathrm{DC}}\right)$ plot with measurements taken at temperature $\mathrm{T}=293 \mathrm{~K}$ and microwave power $\mathrm{P}$ $=16 \mathrm{~mW}$. The $d P / d H$ vs $H_{D C}$ plot exhibited two absorptions which can be associated with two different processes: one centered about $H_{D C}=0$ (LFMA signal) that is more distinct and the other with a ferromagnetic resonant field of $\approx 930$ Oe which is the FMR signal. FMR is due to absorption in the full saturation state. Low-field microwave absorption process usually originates from the magnetization processes far from the saturation state also supported by bulk magnetization shown in Fig 3 (a). The LFMA signal is opposite in phase with respect to that of ferromagnetic resonance. This indicates that the LFMA has microwave absorption minimum at zero field in contrast to the microwave absorption maximum for the FMR line at its resonance field. This is similar to the LFMA in Co-based ribbons as reported by Montiel et al. [8]. LFMA is understood to be connected to the magnetization processes that occur at applied low field. We therefore assume that magnetization processes is present at $\mathrm{H}_{\mathrm{DC}}=0$. Such magnetization process is attributed to the interaction of magnetic moments of thin films of FeSi with the magnetic-field component of electromagnetic radiation. We could not observe hysteresis centered about $H_{D C}=0$ on the $d P / d H$ vs $H_{D C}$ plot due to limitations of the Jeol ESR spectrometer which could not capture the reverse sweep data, though we actually see hysteresis along with the reverse sweep data on the instrument computer screen.

Montiel et al $[8,16]$ have done detailed studies on Co-based amorphous ribbons and alloys and showed a good correlation between the low field hysteresis in magnetization, magneto 
impedance (MI) and LFMA. The observation of LFMA signal at room temperature gives a positive evidence of magnetic state in these films even though the bulk magnetization is very weak at this temperature. In fact Montiel et al [8] show that the peak to peak width of LFMA signal matches very well with that of peak to peak width of MI and corresponds to the anisotropy field. The shape and peak to peak width of LFMA signal also depends on the microwave frequency as per the studies of Lee et al [17] in Co thin films. Lee et al attribute LFMA to the presence of magnetic domains in the unsaturated state of the material. Carara et al [18] show that MI strongly depends on the evolution of domain structure in $\mathrm{FeSi}_{3 \%}$. The fact that LFMA is well correlated with MI and LFMA signal is a positive signature of magnetic state in the material.

To study the role of anisotropy further, we did detailed angular dependence of LFMA on the mutual orientation of $\mathrm{H}_{\mathrm{DC}}$ and the film surface. The microwave magnetic field $\left(\mathrm{H}_{\mathrm{MW}}\right)$ was always parallel to the film. Fig. 4 shows the variation of the derivative of microwave absorption with angle from $\vartheta=0^{\circ}$ to $\vartheta=90^{\circ}$ for the thin film of FeSi. Measurements were carried out at a temperature $\mathrm{T}=293 \mathrm{~K}$ and microwave power $\mathrm{P}=16 \mathrm{~mW}$, while $\mathrm{H}_{\mathrm{MW}}$ was kept parallel to the film surface. At $0^{\circ}$ the intensity of the LFA signal is quite significant and may be due to the induced anisotropy field (IAF) since magnetization remains within the plane. IAF corresponds to the energy needed to orientate the magnetic moments in the easiest direction. When the DC static magnetic field is parallel to the film surface it coincides with easy magnetization axis. A slight difference in the intensity of the LFMA signal is observed between $0^{\circ}$ and $30^{\circ}$ since IAF is still dominant. This therefore implies that magnetic moments are still aligned in the easy axis. The intensity of the signal decreases significantly in the angular variation of $60^{\circ} \leq \vartheta \leq 90^{\circ}$ due to effects of shape anisotropy field (SAF). SAF is maximum at $90^{\circ}$ since magnetization is out of the plane, however at $0^{\circ}$ it is minimum since magnetization remains within the plane [16]. SAF corresponds to the energy needed to orientate magnetic moments in the hardest direction. It can be said that for angular variation of $0^{\circ} \leq \vartheta \leq 90^{\circ}$ both shape anisotropy field (SAF) and induced anisotropy field contribute to total anisotropy field (TAF). Thus TAF is clearly playing a significant role in LFMA in our film and is in good agreement with the scenario given by Montiel et al. $[8,16]$. It should be noted that crystals of ferromagnetic materials and ferrites have easy and difficult directions of magnetization and hence such a strong dependence of LFMA signal on the orientation of DC field with respect to the film surface phenomenon attributes LFMA signal to the ferromagnetic state of a material. 
If this LFMA observed in our film is giving evidence of ferromagnetic state, the long range order should disappear as function of temperature and then LFMA signal should vanish at high temperatures. We show in Fig. 5 the variation of the derivative of the microwave absorption with temperature for the thin film FeSi. Measurements were carried out from temperature $\mathrm{T}=293 \mathrm{~K}$ to $\mathrm{T}=370 \mathrm{~K}$ and microwave power $\mathrm{P}=16 \mathrm{~mW}$. DC field was parallel to the film surface. The LFA spectra is only significant at temperature $\mathrm{T}=293 \mathrm{~K}$ and $\mathrm{T}=313 \mathrm{~K}$. Beyond $\mathrm{T}=313 \mathrm{~K}$, absorption centered at zero field is almost negligible and completely disappears at $340 \mathrm{~K}$. The disappearance of LFMA signal in our film at $340 \mathrm{~K}$ is in good agreement with the arguments of Alvarez et al ${ }^{19}$ that it is indicative of disappearance of long range order and is strongly associated with magnetization processes of the magnetic state. However we do not rule out the origin of LFMA from the formation of ferromagnetic clusters in this film. Such ferromagnetic clusters can disappear at high temperatures and thus lead to the disappearance of LFMA. Either way, LFMA disappearance as a function of temperature indicates its association with some sort of long range order.

Fig. 6 shows the variation of the derivative of the microwave absorption with microwave power for the thin film FeSi. Measurements were carried out from microwave power $\mathrm{P}=3$ $\mathrm{mW}$ to $\mathrm{P}=20 \mathrm{~mW}$ at temperature $\mathrm{T}=293 \mathrm{~K}$. The DC field was parallel to the film surface. LFMA signal showed a significant increase in the intensity of microwave power from $3 \mathrm{~mW}$ to $20 \mathrm{~mW}$ (16 $\mathrm{mW}$ data is not included in this figure for clarity purposes). This shows that microwave power variation has major contribution to the change in the intensity of the absorption spectra. Experimentally as we increase the microwave power, the microwave magnetic field intensity $\mathrm{H}_{M W}$ in the sample cavity increases. The increased $\mathrm{H}_{M W}$ induces more microwave currents on the film surface leading to increased microwave power loss in the film. These are basically the ohmic losses, which are due to impedance. This again is in good agreement with the scenario that LFMA and MI has common origin as described by Montiel et al. [8].

\section{Conclusion}

We have observed LFMA signal for the first time in good quality polycrystalline B20 cubic structure FeSi thin film grown by PLD on Si (111) substrate. The main feature observed in all spectra is that the anisotropy field has a central influence on the LFMA signal strength. Magnetic anisotropy is a signature of ferromagnetic state of a material. The observed LFMA 
signal strongly depends on the mutual orientation of $\mathrm{H}_{\mathrm{DC}}$ and the film surface and disappears at high angles. We qualitatively analyzed these features in terms of total anisotropy. Thus, the observation of LFMA and its strong dependence on the orientation of DC field with respect to film surface gives a positive evidence of magnetic state even at room temperature and above in these film. Further the LFMA signal disappears at temperature above room temperature which proofs the existence of a magnetic state in this film at room temperature. The LFMA signal strength increases with the increase of microwave power, giving additional evidence that the LFMA has common origin with MI as reported in the literature. The observation of LFMA signal in FeSi thin film makes them potential candidates for application as low magnetic field sensors in the microwave and rf frequency regions.

\section{Acknowledgement}

NM acknowledges University of Pretoria research development program and NRF/Ithemba LABS for the financial support of this work.

\section{References}

[1] V. Jaccarino, G. k. Werthem, L. R. Walker, and S. Arajs, Phys. Rev. 160, 476 (1967)

[2] J. H. Wernick, G. K. Werthem and R. C. Sherwood, Mater. Res. Bull. 71431 (1972)

[3] G. Aeppli and J. F. DiTusa, Mater. Sci. Eng. B 63, 119 (1999)

[4] Z. Schlesinger, Z. Fisk, H. -t. Zhang, M. B. Maple, J. F. DiTusa and G. Aeppli, Phys. Rev. Lett. 71, 1748 (1993)

[5] N. Manyala, B. D. Ngom, A. C. Beye, R. Bucker, M. Maaza, A.Strydom, A. Forbes, A. T.Johnson Jr. and J. F. DiTusa, Appl. Phys. Lett.94, 232503 (2009)

[6] S.V. Bhat, P. Ganguly, T.V. Ramakrishnan and C.N.R. Rao, J. Phys.C 20, L559 (1987)

[7] V.V.Srinivasu, S.E. Lofland, S.M.Bhagat, K. Ghosh and S.D. Tyagi, J. Appl. Phys. 86, 1067 (1999).

[8] H. Montiel, G. Alvarez, I. Betancourt, R. Zamorano, R. Valenzuela, Appl. Phys. Lett. 86, $072503(2005)$.

[9] H. Montiel, G. Alvarez, I. Betancourt, R. Zamorano, R. Valenzuela, and R. Zamorano, 
Superficies y Vacio 19, 3 (2006).

[10] F. Owens, J.Phys. Chem. Solids 58, 1311 (1997).

[11] H. Montiel, G. Alvarez, M. Gutierrez, R. Zamorano and R. Valenzuela, J.Alloys Compd. 369, 141 (2004)

[12] G. Alvarez, and R .Zamorano, J. Alloys Comp. 369, 231 (2004).

[13] A. Veinger, A. Zabrodskii and T. Tisnek, Phys. Stat. J. Alloys Comp. 369, 231 (2004).

[14] R. Rakhimov, H. Ries, D .Jones, L. Glebov and L. Glebova, Appl. Phys. Lett. 76, 751 (2000).

[15] G. Alvarez, H. Montiel, D. de Cos, R. Zamorano, A. Garcia-Arribas, J. Barandiaran, and R. Valenzuela, J. Non-Cryst.Solids 353, 902 (2007).

[16] H. Montiel, G. Alvarez, R. Zamorano, and R. Valenzuela, J. Non-Cryst.Solids 353, 908- 910 (2007).

[17]. S.J. Lee, C.C. Tsai, H. Cho, M. Seo, T. Eom, W. Nam, Y.P. Lee and J.B. Ketterson, J. Appl. Phys. 106, 063992 (2009).

[18]. M. Carara, A. Gundel, M.Baibich and R.L. Sommer, J. Appl. Phys. 84, 3792 (1998).

[19]. G. Alvarez, H. Montiel, J.F. Barron, M.P. Gutierrez and R. Zamorano, J.Magn.Magn. mater,322, 348 (2010). 
Fig.1. XRD from bulk and film of FeSi with grown via laser ablation with 15 minutes deposition time.

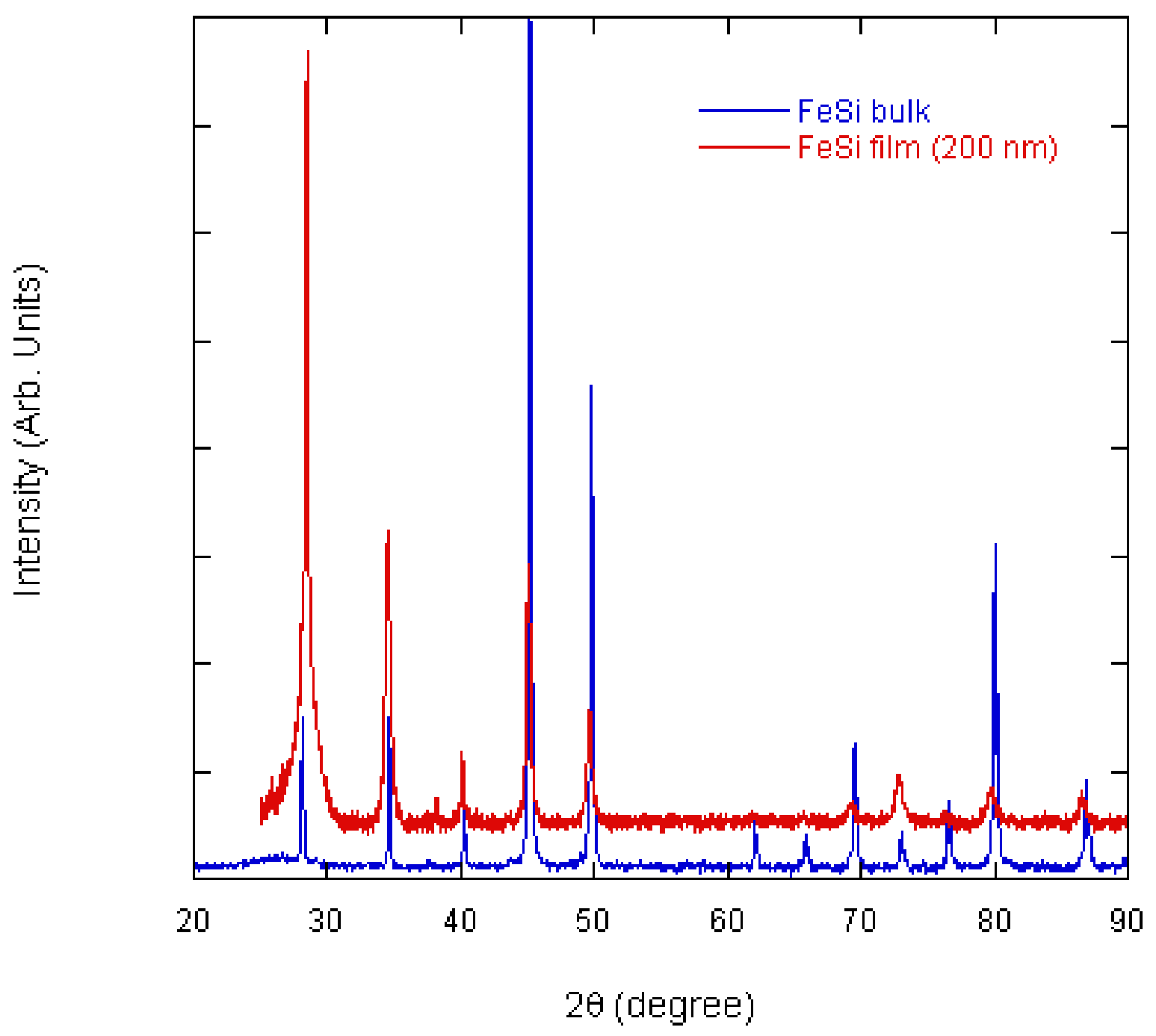


Fig. 2. (a) TEM image [5] and (b) SEM image of FeSi thin film (15 minutes deposition time).
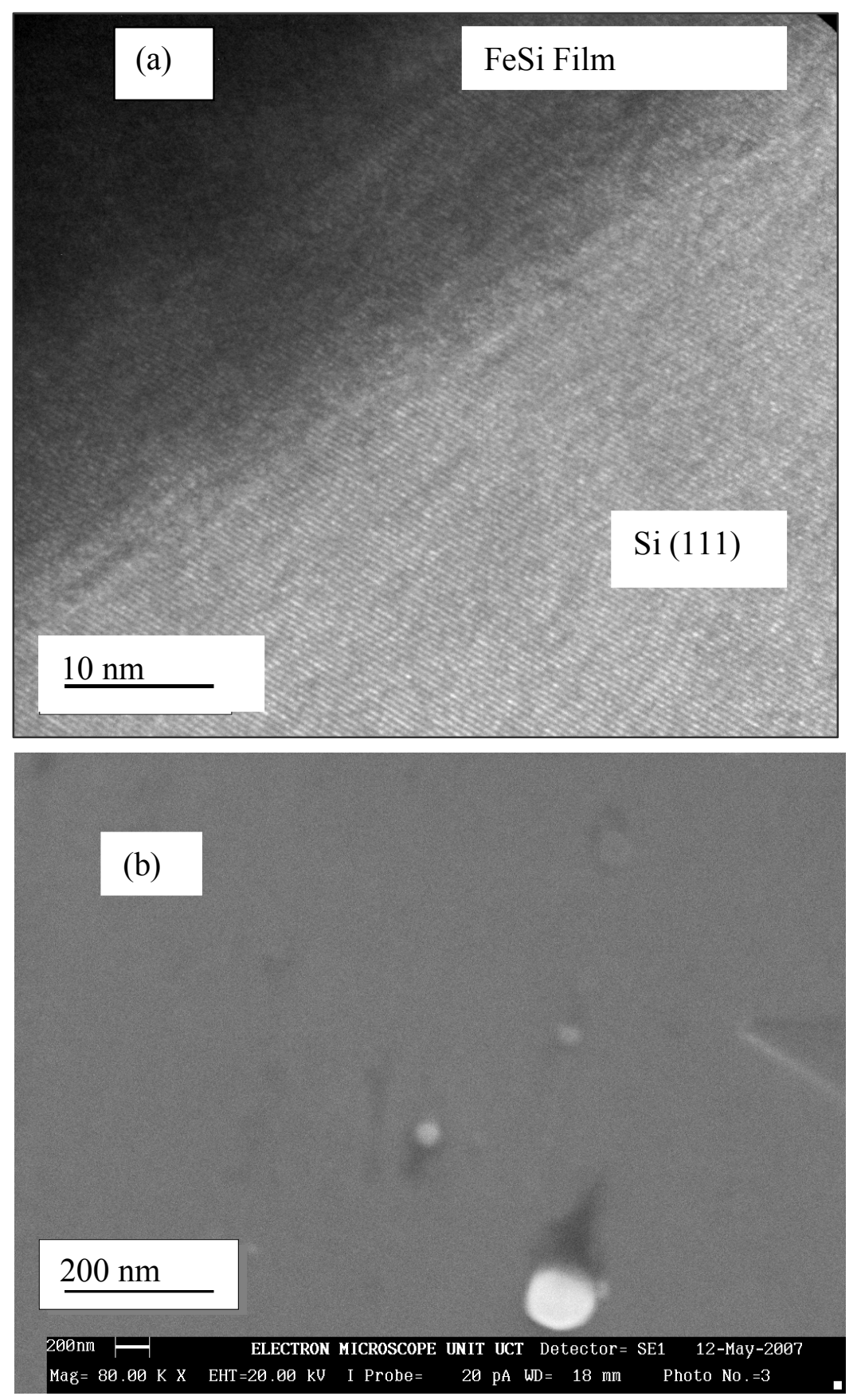


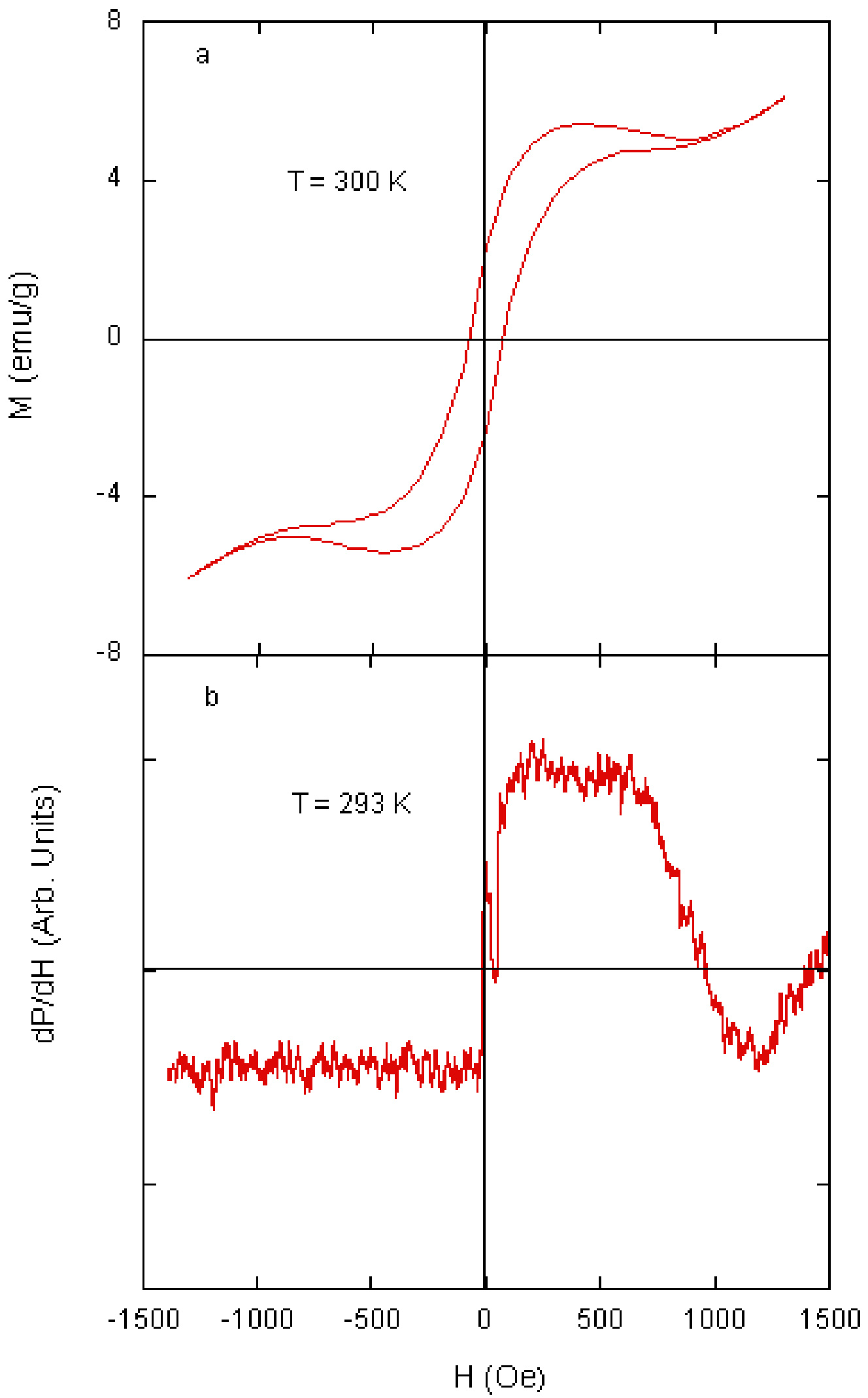

Fig. 3. (a) Bulk hysteresis measurement taken at $\mathrm{T}=300 \mathrm{~K}$, (b) The low field-microwave absorption measurements taken at temperature $T=293 \mathrm{~K}$ with microwave power $\mathrm{P}=16 \mathrm{~mW}$ and DC field parallel to the FeSi film surface. (Note that there is no actual field sweep on the negative direction on the $\mathrm{x}$-axis, due to instrument limitation). 


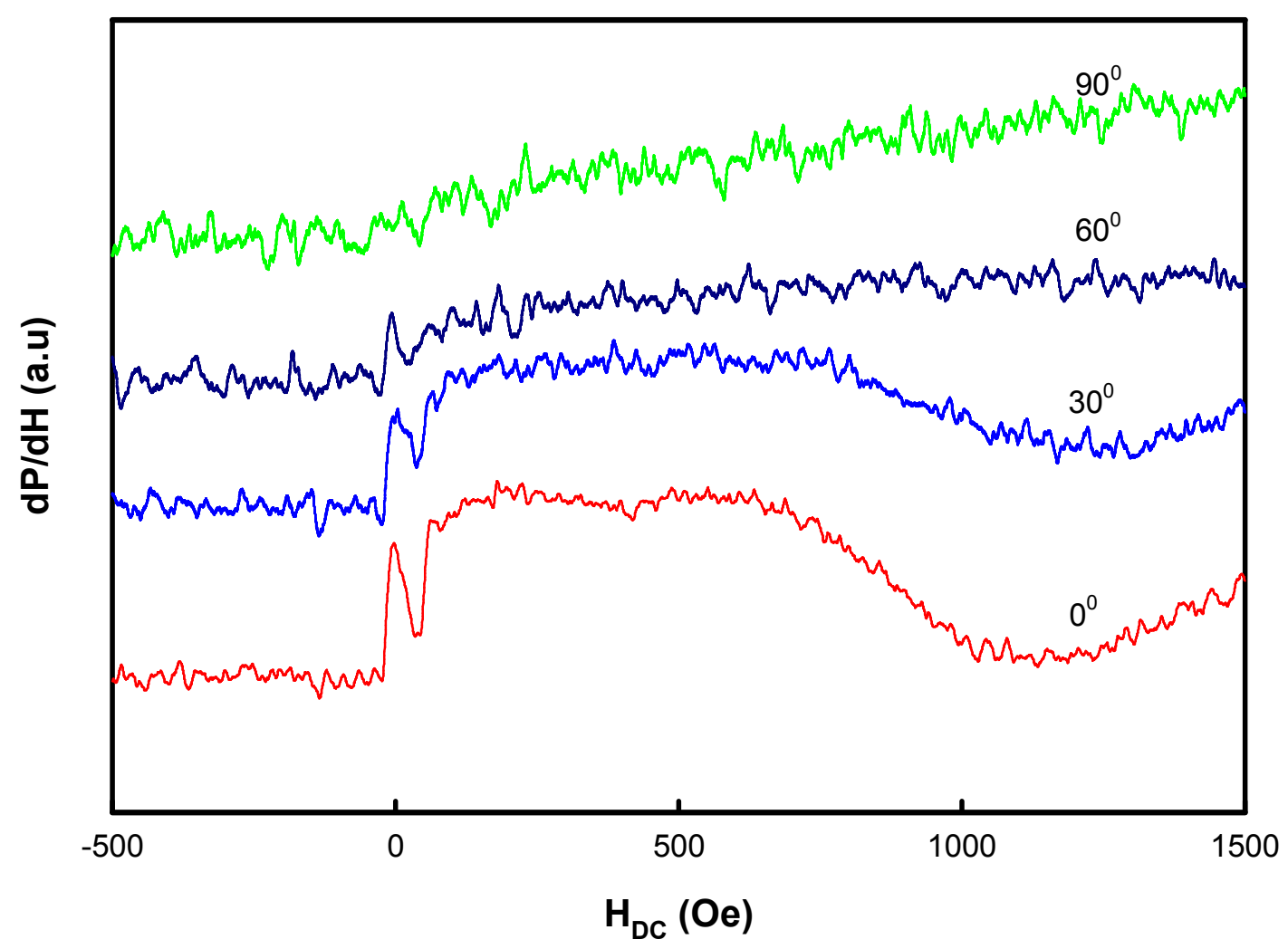

Fig. 4. The angular dependence of low field microwave absorption measurements of FeSi film taken at $\mathrm{T}=293 \mathrm{~K}$ with microwave power of $16 \mathrm{~mW}$. (Note that there is no actual field sweep on the negative direction on the x-axis, due to instrument limitation). 
Fig. 5. The temperature dependence of low field microwave absorption measurements taken at microwave power $\mathrm{P}=16 \mathrm{~mW}$ with $\mathrm{DC}$ field was parallel to the film surface for FeSi film. (Note that there is no actual field sweep on the negative direction on the $\mathrm{x}$-axis, due to instrument limitation).

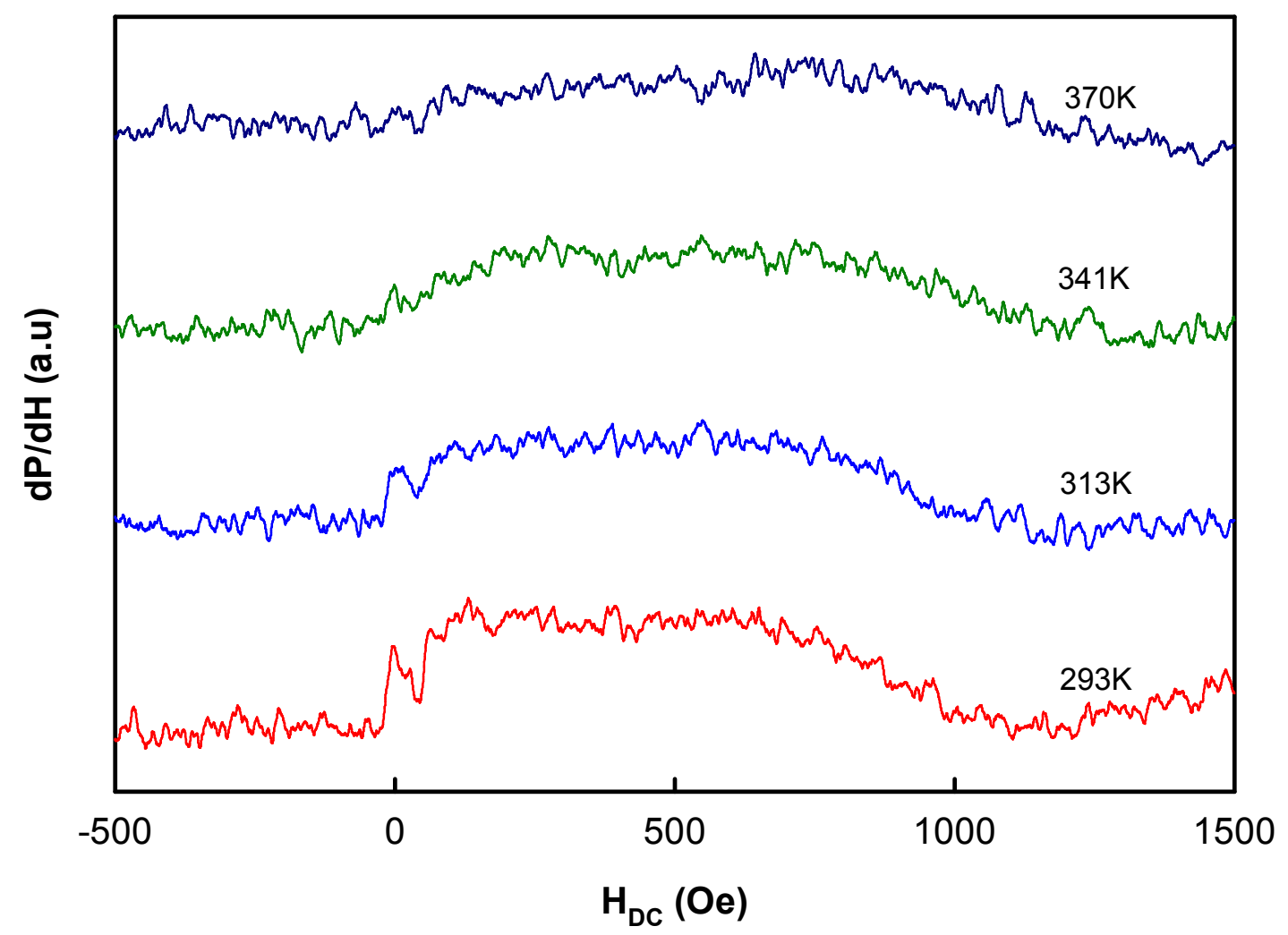


Fig. 6. The microwave power dependence of low field microwave absorption measurements taken at $\mathrm{T}=293 \mathrm{~K}$ with $\mathrm{DC}$ field was parallel to the film surface for FeSi film. (Note that there is no actual field sweep on the negative direction on the $\mathrm{x}$ axis, due to instrument limitation).

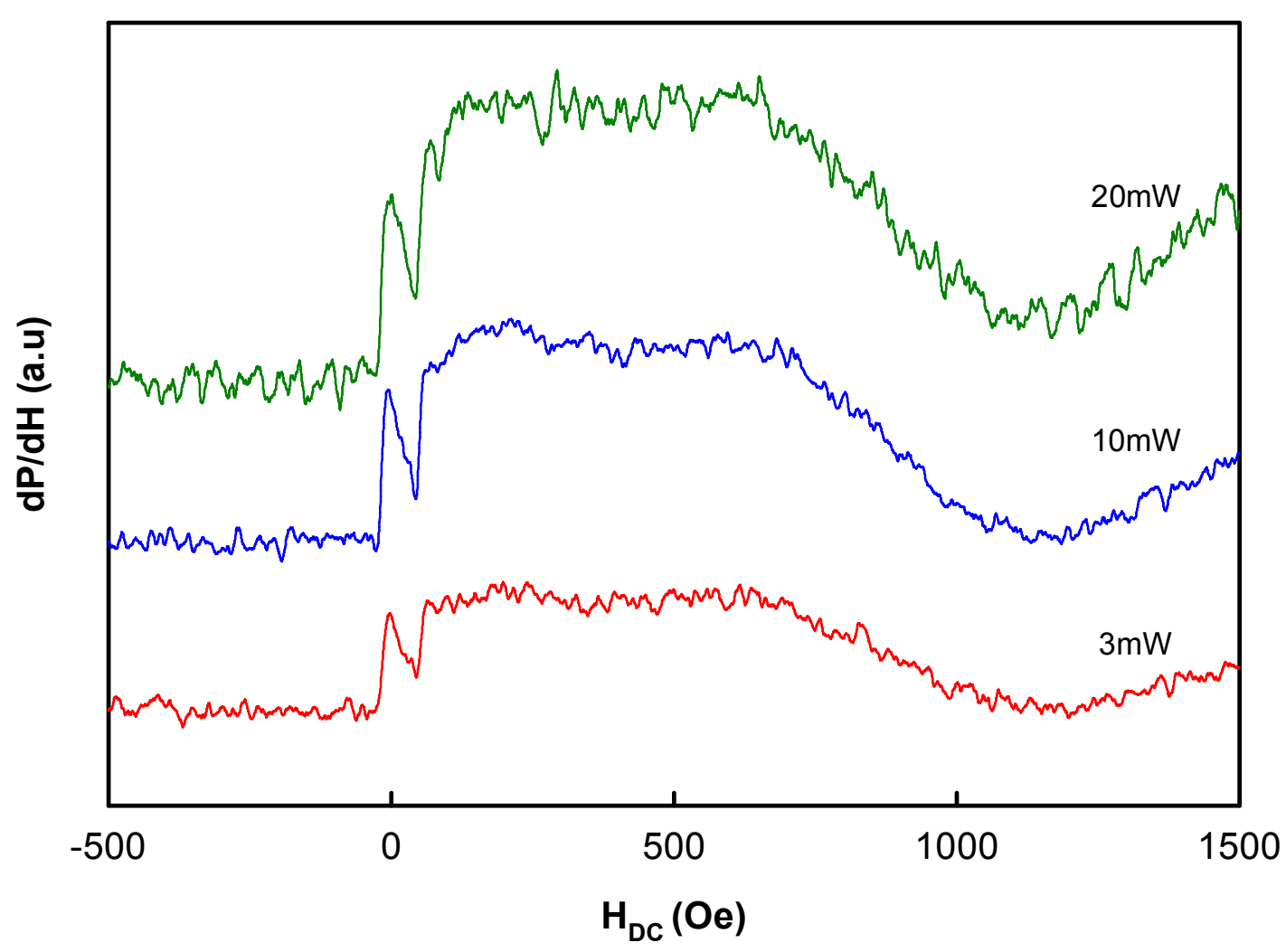

